\title{
ON POWER SERIES HAVING SECTIONS WITH MULTIPLY POSITIVE COEFFICIENTS AND A THEOREM OF PÓLYA
}

\author{
I. V. OSTROVSKII AND N. A. ZHELTUKHINA
}

\section{Introduction}

Let

$$
f(z)=\sum_{k=0}^{\infty} a_{k} z^{k} \quad a_{0}>0
$$

be a formal power series. In 1913, G. Pólya [7] proved that if, for all sufficiently large $n$, the sections

$$
f_{n}(z)=\sum_{k=0}^{n} a_{k} z^{k}
$$

have real negative zeros only, then the series (0.1) converges in the whole complex plane $\mathbf{C}$, and its $\operatorname{sum} f(z)$ is an entire function of order 0 . Since then, formal power series with restrictions on zeros of their sections have been deeply investigated by several mathematicians. We cannot present an exhaustive bibliography here, and restrict ourselves to the references $[\mathbf{1}, \mathbf{2}, \mathbf{3}]$, where the reader can find detailed information.

In this paper, we propose a different kind of generalisation of Pólya's theorem. It is based on the concept of multiple positivity introduced by M. Fekete in 1912, and it has been treated in detail by S. Karlin [4].

Recall that the sequence $\left\{a_{k}\right\}_{k=0}^{\infty}$ of real numbers is said to be $m$-times positive for $m \in \mathbf{N} \cup\{\infty\}$ if all minors of orders less than $m+1$ of the infinite matrix

$$
\left(\begin{array}{ccccc}
a_{0} & a_{1} & a_{2} & a_{3} & \cdots \\
0 & a_{0} & a_{1} & a_{2} & \cdots \\
0 & 0 & a_{0} & a_{1} & \cdots \\
0 & 0 & 0 & a_{0} & \cdots \\
\cdot & \cdot & \cdot & \cdot & \cdots
\end{array}\right)
$$

are non-negative. Usually, $\infty$-times positive sequences are called totally positive sequences.

The Aissen-Edrei-Schoenberg-Whitney theorem (see [4, p. 412]) gives an exhaustive characterisation of totally positive sequences. In particular, this theorem yields the fact that the entire function (0.1) of genus 0 has purely negative zeros if and only if the sequence $\left\{a_{k}\right\}_{k=0}^{\infty}$ is totally positive. Applying this to the polynomial $(0.2)$, we see that negativity of all its zeros is equivalent to total positivity of the sequence $\left\{a_{k}\right\}_{k=0}^{n}:=\left\{a_{0}, a_{1}, \ldots, a_{n}, 0,0,0, \ldots\right\}$. Thus the condition of Pólya's theorem is equivalent to total positivity of the truncated sequences $\left\{a_{k}\right\}_{k=0}^{n}$ for all sufficiently large $n$.

Received 30 June 1995; revised 30 January 1996.

1991 Mathematics Subject Classification 30B10.

J. London Math. Soc. (2) 58 (1998) 97-110 
The following problem seems to be of interest. Does the assertion of Pólya's theorem remain in force if we replace total positivity of the truncated sequences $\left\{a_{k}\right\}_{k=0}^{n}$ by a slower condition of $m$-times positivity for some $m<\infty$ ? It is easy to see that the answer is negative for $m=1$ and $m=2$. For example, if $a_{k}=1$ for any $k=$ $0,1,2, \ldots$, all truncated sequences $\left\{a_{k}\right\}_{k=0}^{n}$ are 2 -times positive, but the series in $(0.1)$ does not converge in the whole plane $\mathbf{C}$. The main aim of this paper is to show that the answer is positive for $m \geqslant 3$. We shall consider some related results and problems.

Note that the $m$-times positivity of the truncated sequence $\left\{a_{k}\right\}_{k=0}^{n}$ for $m<\infty$ is not too closely connected with zeros of the corresponding polynomial (0.2). I. J. Schoenberg (see [9] and [4, pp. 397, 415]) proved that, for $\left\{a_{k}\right\}_{k=0}^{n}, n \geqslant 2$, to be $m$ times positive, the necessary condition is the non-vanishing of $f_{n}(z)$ in the angle $\{z$ : $|\arg z| \leqslant \pi m /(m+n-1)\}$, but the sufficient condition is its non-vanishing in the greater angle $\{z:|\arg z| \leqslant \pi m /(m+1)\}$. Both of the conditions are unimprovable in the sense of the sizes of angles. Note that, for any $m \in \mathbf{N}$, zero-sets of all entire transcendental functions (0.1) with $m$-times positive sequences $\left\{a_{k}\right\}_{k=0}^{\infty}$ form a rather wide class that is described in [5].

\section{Statement of results}

Denote by $P_{m}$ the class of all formal power series $(0.1)$ such that the truncated sequences $\left\{a_{k}\right\}_{k=0}^{n}$ are $m$-times positive for all sufficiently large $n$. Evidently, $P_{m^{\prime}} \subset P_{m}$ for $m^{\prime} \geqslant m$. For any entire function $g(z)$, put $M(r, g)=\max \{|g(z)|:|z| \leqslant r\}$.

THEOREM 1. If a formal power series (0.1) belongs to $P_{m}$ for some $m \geqslant 3$, then it converges in the whole complex plane $\mathbf{C}$, and its sum $f(z)$ is an entire function of order 0. Moreover,

$$
\limsup _{r \rightarrow \infty} \frac{\log M(r, f)}{(\log r)^{2}} \leqslant \frac{1}{2 \log c} \quad c=\frac{1+\sqrt{ } 5}{2} .
$$

The bound (1.1) cannot be improved for $m=3$.

THEOREM 2. There exist entire functions $f(z) \in P_{3}$ such that

$$
\lim _{r \rightarrow \infty} \frac{\log M(r, f)}{(\log r)^{2}}=\frac{1}{2 \log c} .
$$

The question arises of whether the bound (1.1) is unimprovable for $m \geqslant 4$. The consideration of the proof of Pólya's theorem in [7] shows that, in fact, Pólya obtained the following result.

THeORem (Pólya). If a formal power series $(0.1)$ belongs to $P_{\infty}$, then it converges in the whole plane $\mathbf{C}$, and its sum $f(z)$ is an entire function that satisfies the inequality

$$
\limsup _{r \rightarrow \infty} \frac{\log M(r, f)}{(\log r)^{2}} \leqslant \frac{1}{2 \log 2} .
$$

The inequality (1.2) is stronger than (1.1), but we are sure that it is not the best possible even for $f(z) \in P_{4}$. The problem of finding the best possible bound remains open for any $m \geqslant 4$. In this connection, it is worth mentioning that there are entire functions $f(z) \in P_{\infty}$ such that

$$
\lim _{r \rightarrow \infty} \frac{\log M(r, f)}{(\log r)^{2}}=\frac{1}{4 \log 2} .
$$


Such a function,

$$
f(z)=\sum_{k=0}^{\infty} 2^{-k^{2}} z^{k}
$$

was considered in [8, Problem 176, p. 66]. Hence the unknown best possible bound (probably depending on $m$ ) is not less than $1 /(4 \log 2)$.

Denote by $Q_{m}$ the subclass of $P_{m}$ consisting of formal power series $(0.1)$ satisfying the following condition: for all $n=0,1,2, \ldots$, the truncated sequences $\left\{a_{k}\right\}_{k=0}^{n}$ are $m$ times positive. For $f(z) \in Q_{m}$, the bound (1.1) can be improved in the following way.

THEOREM 3. If a formal power series $(0.1)$ belongs to $Q_{m}$ for some $m \geqslant 3$, then the following refinement of (1.1) is valid:

$$
M(r, f) \leqslant a_{0} \vartheta_{3}\left(0, \frac{1}{\sqrt{c}}\right) \exp \left\{\frac{\left(\log \left(r \sqrt{c} a_{1} / a_{0}\right)\right)^{2}}{2 \log c}\right\}
$$

where $\vartheta_{3}$ denotes the Jacobi theta-function $([\mathbf{1 0}, 21.11$, p. 464]). Under the normalisation condition $a_{0}=a_{1}=1$, (1.3) takes a simpler form:

$$
M(r, f) \leqslant \vartheta_{3}\left(0, \frac{1}{\sqrt{c}}\right) c^{1 / 8} \sqrt{r} \exp \left\{\frac{(\log r)^{2}}{2 \log c}\right\} .
$$

COROLlary 1. If $m$ is larger than or equal to 3 , then the set of all entire functions $f(z) \in Q_{m}$ with fixed coefficients $a_{0}>0, a_{1}$ is a normal family.

The inequalities (1.3) and (1.4) are not sharp, at least for small $r$, since their righthand sides tend to $+\infty$ as $r$ tends to 0 . Now we are going to obtain a bound which is more complicated, but the best possible for all $r \geqslant 0$ and all $f(z) \in Q_{3}$.

Let $\left\{z_{k}\right\}_{k=2}^{\infty}$ be the sequence of positive numbers defined by the recurrence equation

$$
z_{k+1}^{2}=z_{k}+1 \quad k=2,3, \ldots
$$

and by the initial condition

$$
z_{2}=1
$$

Define the following:

$$
\begin{gathered}
d_{k}=1+\frac{1}{z_{k}} \quad k=2,3, \ldots \\
(z)=1+z+\sum_{k=2}^{\infty} \frac{z^{k}}{d_{2}^{k-1} d_{2}^{k-2} \ldots d_{k}} .
\end{gathered}
$$

THEOREM 4. If a formal power series (0.1) belongs to $Q_{m}$ for some $m \geqslant 3$, then the following inequality is valid:

$$
M(r, f) \leqslant a_{0} \quad\left(a_{1} r / a_{0}\right) \quad r \geqslant 0 .
$$

A. Edrei [1] proved that, if each $f_{n}(z)$ does not vanish in some half-plane (possibly, depending on $n)$, then the series $(0.1)$ converges in the whole plane and its sum satisfies the condition

$$
\log M(r, f)=O\left((\log r)^{2}\right) \quad r \rightarrow \infty
$$


T. Ganelius [3] proved that (1.10) remains in force if the half-plane is replaced by any angle of positive size not depending on $n$. As a corollary of Theorem 1, we obtain the following Theorem 5, which sharpens the bound (1.10) under some additional conditions.

THEOREM 5. Let $f(z)$ be a formal power series of the form (0.1) with real coefficients $a_{k}$. Assume that, for sufficiently large $n$, the zeros of the sections (0.2) are located in the angle $\{z:|\arg z-\pi|<\alpha\}$. Then the series converges in the whole plane $\mathbf{C}$, and, moreover,

(i) if $\alpha$ is smaller than or equal to $\pi / 4$, then (1.1) is valid;

(ii) if $\alpha$ is smaller than or equal to $\pi / 2$, then

is valid.

$$
\limsup _{r \rightarrow \infty} \frac{\log M(r, f)}{(\log r)^{2}} \leqslant \frac{2}{\log 2}
$$

\section{Proof of Theorem 1}

Henceforth, we assume that the series (0.1) contains infinitely many non-zero terms; otherwise, the statement of Theorem 1 is trivial. Under this assumption, the sequence $\left\{a_{k}\right\}_{k=0}^{\infty}$ cannot contain zero terms at all, as the following (known) Lemma 1 shows.

Lemma 1. Let $\left\{a_{k}\right\}_{k=0}^{\infty}, a_{0}>0$, be a 2-times positive sequence. Set $n=\min \{k$ : $\left.a_{k}=0\right\}$. If $n$ is finite, then $a_{k}=0$ for any $k \geqslant n$.

Proof. By the definition of 2-times positivity, we have, for any $k>n$,

$$
\left|\begin{array}{ll}
a_{n} & a_{k} \\
a_{n-1} & a_{k-1}
\end{array}\right| \geqslant 0 .
$$

Since $a_{n}=0, a_{n-1}>0$, we conclude that $a_{k}=0$.

Evidently, 3-times positivity of truncated sequences $\left\{a_{k}\right\}_{k=0}^{n}$ for sufficiently large $n$ yields 3 -times positivity of the whole sequence $\left\{a_{k}\right\}_{k=0}^{\infty}$. Hence, Lemma 1 is applicable, and all the $a_{k}$ are strictly positive. This allows us to introduce the positive numbers

It is evidence that

$$
\rho_{k}=\frac{a_{k-1}}{a_{k}} \quad k=1,2, \ldots
$$

$$
a_{k}=\frac{a_{0}}{\prod_{j=1}^{k} \rho_{j}} \quad k=1,2, \ldots
$$

The following (known) Lemma 2 shows that the numbers $\rho_{k}$ form a non-decreasing sequence.

Lemma 2. Let $\left\{a_{k}\right\}_{k=0}^{\infty}$ be a 2-times positive sequence without zero terms. Then the sequence $\left\{\rho_{k}\right\}_{k=1}^{\infty}$ defined by (2.1) is non-decreasing.

Proof. The inequality

is equivalent to $\rho_{k} \leqslant \rho_{k+1}$.

$$
\left|\begin{array}{ll}
a_{k} & a_{k+1} \\
a_{k-1} & a_{k}
\end{array}\right| \geqslant 0
$$


Define the numbers as follows:

Evidently,

$$
\delta_{k}=\frac{\rho_{k}}{\rho_{k-1}} \quad k=2,3, \ldots
$$

By Lemma 2, we have

$$
\rho_{k}=\rho_{1} \prod_{j=2}^{k} \delta_{j} \quad k=2,3, \ldots
$$

Using (2.2), we obtain

$$
\delta_{k} \geqslant 1 \quad k=2,3, \ldots
$$

$$
a_{k}=\frac{a_{0}}{\rho_{1}^{k} \delta_{2}^{k-1} \delta_{3}^{k-2} \ldots \delta_{k}} \quad k=1,2,3, \ldots
$$

The following Lemma 3 plays a basic role in the proof of Theorem 1.

Lemma 3. Let $\left\{a_{k}\right\}_{k=0}^{n}=\left\{a_{0}, a_{1}, \ldots, a_{n}, 0,0, \ldots\right\}, a_{0}>0, a_{n}>0, n \geqslant 2$, be a 3-times positive sequence. Then

(i) for $n=2$, we have $\delta_{2} \geqslant 2$;

(ii) for $n \geqslant 3$, we have $\delta_{n}>1$ and

$$
\left(\delta_{n}-1\right)^{2} \geqslant 1-\frac{1}{\delta_{n-1}} .
$$

Proof. If $n=2$, we have

$$
\left|\begin{array}{lll}
a_{1} & a_{2} & 0 \\
a_{0} & a_{1} & a_{2} \\
0 & a_{0} & a_{1}
\end{array}\right| \geqslant 0 .
$$

Using this and (2.4), we obtain $\delta_{2} \geqslant 2$.

If $n \geqslant 3$, we have

$$
\left|\begin{array}{lll}
a_{1} & a_{n} & 0 \\
a_{0} & a_{n-1} & a_{n} \\
0 & a_{n-2} & a_{n-1}
\end{array}\right| \geqslant 0 .
$$

Calculating the determinant, we obtain

$$
a_{1} a_{n-1}^{2}-a_{0} a_{n-1} a_{n}-a_{1} a_{n-2} a_{n}=a_{1} a_{n-2} a_{n}\left(\frac{a_{n-1}^{2}}{a_{n-2} a_{n}}-\frac{a_{0} a_{n-1}}{a_{1} a_{n-2}}-1\right) \geqslant 0 .
$$

Since (2.4) yields

we get

$$
\frac{a_{n-1}^{2}}{a_{n-2} a_{n}}=\delta_{n} \quad \frac{a_{0} a_{n-1}}{a_{1} a_{n-2}}=\frac{1}{\delta_{2} \ldots \delta_{n-1}},
$$

Further, we have

$$
\delta_{n} \geqslant 1+\frac{1}{\delta_{2} \ldots \delta_{n-1}}>1
$$

$$
\left|\begin{array}{lll}
a_{n-1} & a_{n} & 0 \\
a_{n-2} & a_{n-1} & a_{n} \\
a_{n-3} & a_{n-2} & a_{n-1}
\end{array}\right| \geqslant 0 .
$$


Calculating the determinant, we obtain

$$
a_{n-1}^{3}+a_{n-3} a_{n}^{2}-2 a_{n-2} a_{n-1} a_{n}=a_{n-3} a_{n}^{2}\left(\frac{a_{n-1}^{3}}{a_{n-3} a_{n}^{2}}+1-2 \frac{a_{n-2} a_{n-1}}{a_{n-3} a_{n}}\right) \geqslant 0 .
$$

Since (2.4) yields

we get

$$
\frac{a_{n-1}^{3}}{a_{n-3} a_{n}^{2}}=\delta_{n}^{2} \delta_{n-1} \quad \frac{a_{n-2} a_{n-1}}{a_{n-3} a_{n}}=\delta_{n} \delta_{n-1},
$$

$$
\delta_{n}^{2} \delta_{n-1}+1-2 \delta_{n} \delta_{n-1} \geqslant 0 .
$$

This inequality is equivalent to (2.5).

If a formal power series (0.1) satisfies the condition of Theorem 1 , then there exists some $n_{0} \geqslant 2$ such that, for each $n \geqslant n_{0}$, the truncated sequence $\left\{a_{k}\right\}_{k=0}^{n}$ is 3 -times positive. By Lemma 3, we have $\delta_{n}>1$ for $n \geqslant n_{0}$, and therefore the numbers

$$
y_{n}:=\frac{1}{\delta_{n}-1} \quad n \geqslant n_{0}
$$

are well defined. Using (2.5), we obtain

$$
y_{n+1}^{2} \leqslant y_{n}+1 \quad n \geqslant n_{0} .
$$

Consider the sequence $\left\{z_{n}\right\}_{n=n_{0}}^{\infty}$ of positive numbers satisfying the recurrence equation

and the initial condition

$$
z_{n+1}^{2}=z_{n}+1 \quad n \geqslant n_{0}
$$

It is easy to see that

$$
z_{n_{0}}=y_{n_{0}} .
$$

$$
y_{n} \leqslant z_{n} \quad n \geqslant n_{0} .
$$

LEMma 4. There exists the limit

$$
\lim _{n \rightarrow \infty} z_{n}=\frac{1+\sqrt{5}}{2}=c .
$$

Moreover,

(i) if $z_{n_{0}}$ is smaller than $c$, then the sequence $\left\{z_{n}\right\}_{n=n_{0}}^{\infty}$ increases;

(ii) if $z_{n_{0}}$ is larger than $c$, then the sequence $\left\{z_{n}\right\}_{n=n_{0}}^{\infty}$ decreases;

(iii) if $z_{n_{0}}=c$, then $z_{n}=c$ for any $n \geqslant n_{0}$.

Proof. The proof of Lemma 4 is based on the fact that $c$ is a root of the equation $z^{2}=z+1$, and $z^{2}<z+1$ for $0 \leqslant z<c$ and $z^{2}>z+1$ for $z>c$. The details can be omitted.

Using (2.6), (2.10) and Lemma 4, we obtain

$$
\liminf _{n \rightarrow \infty} \delta_{n}=\liminf _{n \rightarrow \infty}\left(1+\frac{1}{y_{n}}\right) \geqslant \lim _{n \rightarrow \infty}\left(1+\frac{1}{z_{n}}\right)=1+\frac{1}{c}=c .
$$

If $0<\varepsilon<c-1$, then we have

$$
\delta_{n} \geqslant c-\varepsilon \quad n>q=q(\varepsilon) .
$$


By (2.4), we obtain, for $k>q$,

$$
\begin{aligned}
\log a_{k} & =\log a_{0}-k \log \rho_{1}-\sum_{j=2}^{k}(k-j+1) \log \delta_{j} \\
& \leqslant \log a_{0}-k \log \rho_{1}-\sum_{j=2}^{q}(k-j+1) \log \delta_{j}-\sum_{j=q+1}^{k}(k-j+1) \log (c-\varepsilon) \\
& =-\frac{k^{2}}{2} \log (c-\varepsilon)+O(k) \quad k \rightarrow \infty .
\end{aligned}
$$

Hence,

$$
a_{k} \leqslant C D^{k}(c-\varepsilon)^{-k^{2} / 2} \quad k=0,1,2, \ldots,
$$

where $C$ and $D$ are positive constants not depending on $k$. Since $c-\varepsilon$ is larger than 1 , (2.13) yields the fact that $\lim _{k \rightarrow \infty} a_{k}^{1 / k}=0$. Hence the series $(0.1)$ converges in the whole plane.

Further, using (2.13), we have

$$
\begin{aligned}
M(r, f) & =f(r)=\sum_{k=0}^{\infty} a_{k} r^{k} \leqslant C \sum_{k=0}^{\infty}(c-\varepsilon)^{-k^{2} / 2}(D r)^{k} \\
& =C \exp \left(\frac{(\log D r)^{2}}{2 \log (c-\varepsilon)}\right) \sum_{k=0}^{\infty} \exp \left\{-\frac{\log (c-\varepsilon)}{2}\left(k-\frac{\log (D r)}{\log (c-\varepsilon)}\right)^{2}\right\} \\
& <C \exp \left(\frac{(\log D r)^{2}}{2 \log (c-\varepsilon)}\right) \sup _{-\infty<x<\infty} \sum_{k=-\infty}^{\infty} \exp \left\{-\frac{\log (c-\varepsilon)}{2}(k-x)^{2}\right\} .
\end{aligned}
$$

Since the sum of the series under the supremum sign is a periodic function of $x$ (with period 1), its supremum is finite. Hence

and

$$
M(r, f)=O\left(\exp \left\{\frac{(\log D r)^{2}}{2 \log (c-\varepsilon)}\right\}\right) \quad r \rightarrow \infty
$$

$$
\log M(r, f) \leqslant \frac{(\log r)^{2}}{2 \log (c-\varepsilon)}+O(\log r) \quad r \rightarrow \infty .
$$

Since $\varepsilon>0$ is arbitrary, we obtain the inequality (1.1).

Proof of Pólya's theorem. Now we present the proof of Pólya's theorem for the reader's convenience. The condition $f(z) \in P_{\infty}$ means (see the introduction) that the sections ( 0.2$)$ have purely negative zeros for sufficiently large $n$, that is, for $n \geqslant n_{1}$ say. It is well known that derivatives of a polynomial having purely real zeros have purely real zeros. Hence, the quadratic polynomial

$$
\left(\frac{d}{d z}\right)^{n-2} f_{n}(z)=(n-2) ! a_{n-2}+(n-1) ! a_{n-1} z+\frac{1}{2} n ! a_{n} z^{2}
$$

does not have any complex zero. This means that the following inequality is valid:

$$
(n-1) a_{n-1}^{2} \geqslant 2 n a_{n-2} a_{n} \quad n \geqslant n_{1} .
$$

Remembering the definition of $\delta_{n}$, we can rewrite the last inequality in the form

$$
\delta_{n} \geqslant \frac{2 n}{n-1}>2 \quad n \geqslant n_{1} .
$$


Therefore (2.11) is valid with 2 in place of $c-\varepsilon$. This yields (2.12) and (2.13) with 2 instead of $c-\varepsilon$.

\section{Proofs of Theorems 3 and 4}

Proof of Theorem 3. If $f(z)$ belongs to $Q_{3}$, then, by Lemma 3(i), we have $\delta_{2} \geqslant$ 2 , and, moreover, we can take $n_{0}=2$ in (2.7)-(2.10). Since $z_{2}=y_{2} \leqslant 1<c$, Lemma 4 yields the fact that the sequence $\left\{z_{n}\right\}_{n=2}^{\infty}$ is increasing and $z_{n}$ is smaller than $c$ for $n$ $\geqslant 2$. Hence $y_{n}<c$, and

$$
\delta_{n}=1+\frac{1}{y_{n}}>1+\frac{1}{c}=c \quad n \geqslant 2 .
$$

Using this, we can improve (2.12) in the following way:

$$
\begin{aligned}
\log a_{k} & =\log a_{0}-k \log \rho_{1}-\sum_{j=2}^{k}(k-j+1) \log \delta_{j} \\
& \leqslant \log a_{0}-k \log \rho_{1}-\frac{k(k-1)}{2} \log c \quad k \geqslant 2 .
\end{aligned}
$$

We obtain the following refinement of (2.13):

Hence

$$
a_{k} \leqslant a_{0}\left(\sqrt{c} / \rho_{1}\right)^{k} c^{-k^{2} / 2} \quad k=0,1,2, \ldots
$$

$$
\begin{aligned}
M(r, f) & \leqslant a_{0} \sum_{k=0}^{\infty} c^{-k^{2} / 2}\left(r \sqrt{c} / \rho_{1}\right)^{k} \\
& =a_{0} \exp \left\{\frac{\left(\log \left(r \sqrt{c} / \rho_{1}\right)\right)^{2}}{2 \log c}\right\} \sum_{k=0}^{\infty} \exp \left\{-\frac{\log c}{2}\left(k-\frac{\log \left(r \sqrt{c} / \rho_{1}\right)}{\log c}\right)^{2}\right\} \\
& \leqslant a_{0} \exp \left\{\frac{\left(\log \left(r \sqrt{c} / \rho_{1}\right)\right)^{2}}{2 \log c}\right\} \sup _{-\infty<x<\infty} \sum_{k=-\infty}^{\infty} \exp \left\{-\frac{\log c}{2}(k-x)^{2}\right\} .
\end{aligned}
$$

By a well known formula of the theory of theta-functions [10, 21.51, p. 476], we have, for any $\alpha>0$,

$$
\sum_{k=-\infty}^{\infty} \exp \left(-\frac{\pi^{2} k^{2}}{\alpha}+2 \pi k \mathrm{i} x\right)=\frac{\bar{\alpha}}{\pi} \sum_{k=-\infty}^{\infty} \exp \left(-\alpha(k-x)^{2}\right)
$$

Hence the sum of the series in the right-hand side attains its maximal value when $x=0$, and we obtain

$$
\sup _{-\infty<x<\infty} \sum_{k=-\infty}^{\infty} \exp \left(-\frac{\log c}{2}(k-x)^{2}\right)=\sum_{k=-\infty}^{\infty} \exp \left(-\frac{\log c}{2} k^{2}\right)=\vartheta_{3}\left(0, \frac{1}{\sqrt{c}}\right) .
$$

Remark 1. If $f(z)$ belongs to $Q_{\infty}$, then (1.3) and (1.4) can be improved by the replacement of $c$ by 2 .

Indeed, the condition $f(z) \in Q_{\infty}$ yields the fact that the inequality (2.14) is valid for any $n \geqslant 2$. Using this inequality instead of (3.1), we obtain the claimed result.

Proof of Theorem 4. By Lemma 3, we have $\delta_{2} \geqslant 2$, and the numbers $y_{n}$ are well defined by (2.6) for all $n \geqslant 2$. In particular, we have

$$
y_{2}=\frac{1}{\delta_{2}-1} \leqslant 1=z_{2} .
$$


By (2.10) with $n_{0}=2$, we have $y_{k} \leqslant z_{k}$ for any $n \geqslant 2$. Therefore,

$$
\delta_{k}=1+\frac{1}{y_{k}} \geqslant 1+\frac{1}{z_{k}}=d_{k} \quad k \geqslant 2 .
$$

Using (2.4) and (1.8), we obtain

$$
\begin{aligned}
M(r, f) & =a_{0}+a_{1} r+\sum_{k=2}^{\infty} \frac{a_{0} r^{k}}{\rho_{1}^{k} \delta_{2}^{k-1} \ldots \delta_{k}} \\
& \leqslant a_{0}+a_{1} r+\sum_{k=2}^{\infty} \frac{a_{0} r^{k}}{\rho_{1}^{k} d_{2}^{k-1} \ldots d_{k}}=a_{0} \quad\left(\frac{a_{1}}{a_{0}} r\right) .
\end{aligned}
$$

ReMARK 2. If $f(z)$ belongs to $Q_{\infty}$, then the inequality (1.9) can be replaced by the following more precise

where

$$
M(r, f) \leqslant a_{0} \psi\left(a_{1} r / a_{0}\right)
$$

$$
\psi(z)=1+z+\sum_{k=2}^{\infty} \frac{z^{k}}{2^{k(k-1) / 2} k !} .
$$

Indeed, since (2.14) is valid for any $n \geqslant 2$, we have

$$
\rho_{n}=\delta_{n} \rho_{n-1} \geqslant \frac{2 n}{n-1} \rho_{n-1} \geqslant \ldots \geqslant 2^{n-1} n \rho_{1} .
$$

Hence, using (2.2), we obtain

$$
\begin{aligned}
M(r, f) & =a_{0}+a_{1} r+\sum_{n=2}^{\infty} \frac{a_{0} r^{n}}{\rho_{1} \rho_{2} \ldots \rho_{n}} \\
& \leqslant a_{0}+a_{1} r+\sum_{n=2}^{\infty} \frac{a_{0} r^{n}}{\rho_{1}^{n} 2^{n(n-1) / 2} n !}=a_{0} \psi\left(\frac{a_{1}}{a_{0}} r\right) .
\end{aligned}
$$

Note that inequality (3.3) is contained in an implicit form in [7].

\section{Proof of Theorem 2}

Lemma 5. The function $(z)$ defined by (1.8) belongs to $Q_{3} \subset P_{3}$.

Proof. We shall use the following test of $m$-times positivity.

THEOREM (I. J. Schoenberg [9]). Let $\left\{b_{k}\right\}_{k=0}^{n}$ be a finite sequence of numbers. Consider $m$ matrices

$$
B_{k}=\left(\begin{array}{lllllllll}
b_{0} & b_{1} & b_{2} & \cdots & b_{n} & 0 & 0 & \cdots & 0 \\
0 & b_{0} & b_{1} & \cdots & b_{n-1} & b_{n} & 0 & \cdots & 0 \\
0 & 0 & b_{0} & \cdots & b_{n-2} & b_{n-1} & b_{n} & \cdots & 0 \\
\cdot & \cdot & \cdot & \cdots & \cdot & \cdot & \cdot & \cdots & \cdot \\
0 & 0 & 0 & \cdots & \cdot & \cdot & \cdot & \cdots & b_{n}
\end{array}\right) \quad k=1,2, \ldots, m,
$$

where $B_{k}$ consists of $k$ rows and $n+k$ columns. Assume that the following condition is satisfied for $k=1,2, \ldots, m$ : all $k \times k$-minors of $B_{k}$ consisting of consecutive columns are strictly positive. Then the sequence $\left(b_{0}, b_{1}, \ldots, b_{n}, 0,0, \ldots\right\}$ is m-times positive. 
Let

$$
a_{0}=a_{1}=1 \quad a_{k}=\frac{1}{d_{2}^{k-1} d_{3}^{k-2} \ldots d_{k}} \quad k=2,3, \ldots
$$

be the coefficients of the function $(z)$. Fix any $n \geqslant 2$, and consider the sequence

$$
\left\{a_{0}, a_{1}, \ldots, a_{n-1}, a_{n}-\varepsilon, 0,0, \ldots\right\},
$$

where $\varepsilon>0$ will be chosen sufficiently small later. Form three matrices:

$$
\begin{gathered}
A_{1}=\left(\begin{array}{lllllll}
a_{0} & a_{1} & \ldots & a_{n-1} & a_{n}-\varepsilon
\end{array}\right) \\
A_{2}=\left(\begin{array}{llllllll}
a_{0} & a_{1} & a_{2} & \ldots & a_{n-1} & a_{n}-\varepsilon & 0 \\
0 & a_{0} & a_{1} & \ldots & a_{n-2} & a_{n-1} & a_{n}-\varepsilon
\end{array}\right) \\
A_{3}=\left(\begin{array}{llllllll}
a_{0} & a_{1} & a_{2} & \ldots & a_{n-1} & a_{n}-\varepsilon & 0 & 0 \\
0 & a_{0} & a_{1} & \ldots & a_{n-2} & a_{n-1} & a_{n}-\varepsilon & 0 \\
0 & 0 & a_{0} & \ldots & a_{n-3} & a_{n-2} & a_{n-1} & a_{n}-\varepsilon
\end{array}\right) .
\end{gathered}
$$

All minors of $A_{1}$ are trivially positive for $0<\varepsilon<a_{n}$. Since

$$
\frac{a_{k}}{a_{k+1}}=d_{2} d_{3} \ldots d_{k+1} \quad k=1,2, \ldots
$$

and $d_{k}>c>1$, we have

$$
\frac{a_{k-1}}{a_{k}}<\frac{a_{k}}{a_{k+1}} \quad k=1,2, \ldots, n-2
$$

and

$$
\frac{a_{n-2}}{a_{n-1}}<\frac{a_{n-1}}{a_{n}-\varepsilon}
$$

for sufficiently small $\varepsilon$. Therefore, all minors of $A_{2}$ are positive for such $\varepsilon$.

Further, consider the determinants

We have

$$
N_{k}=\left|\begin{array}{lll}
a_{k} & a_{k+1} & 0 \\
a_{k-1} & a_{k} & a_{k+1} \\
a_{k-2} & a_{k-1} & a_{k}
\end{array}\right| \quad k=2,3, \ldots
$$

$$
\begin{aligned}
N_{k}=a_{k}^{3}+a_{k+1}^{2} a_{k-2}-2 a_{k+1} a_{k} a_{k-1} & =a_{k+1}^{2} a_{k-2}\left(d_{k+1}^{2} d_{k}+1-2 d_{k+1} d_{k}\right) \\
& =a_{k+1}^{2} a_{k-2} d_{k}\left(\frac{1}{z_{k+1}^{2}}-\frac{1}{z_{k}+1}\right)=0 \quad k=2,3, \ldots
\end{aligned}
$$

by virtue of (4.1), (1.7) and (1.5). Moreover, setting

$$
N_{1}=\left|\begin{array}{ccc}
a_{1} & a_{2} & 0 \\
a_{0} & a_{1} & a_{2} \\
0 & a_{0} & a_{1}
\end{array}\right|=1-\frac{2}{d_{2}},
$$

we have $N_{1}=0$, since $d_{2}=2$ by virtue of (1.7) and (1.6). 
Now, consider the $3 \times 3$-minors of $A_{3}$ consisting of consecutive columns:

$$
\begin{gathered}
M_{0}=\left|\begin{array}{lll}
a_{0} & a_{1} & a_{2} \\
0 & a_{0} & a_{1} \\
0 & 0 & a_{0}
\end{array}\right| \quad M_{1}=\left|\begin{array}{lll}
a_{1} & a_{2} & a_{3} \\
a_{0} & a_{1} & a_{2} \\
0 & a_{0} & a_{1}
\end{array}\right| \\
M_{k}=\left|\begin{array}{lll}
a_{k} & a_{k+1} & a_{k+2} \\
a_{k-1} & a_{k} & a_{k+1} \\
a_{k-2} & a_{k-1} & a_{k}
\end{array}\right| \quad k=2,3, \ldots, n-3 \\
M_{n-2}(\varepsilon)=\left|\begin{array}{lll}
a_{n-2} & a_{n-1} & a_{n}-\varepsilon \\
a_{n-3} & a_{n-2} & a_{n-1} \\
a_{n-4} & a_{n-3} & a_{n-2}
\end{array}\right| M_{n-1}(\varepsilon)=\left|\begin{array}{lll}
a_{n-1} & a_{n}-\varepsilon & 0 \\
a_{n-2} & a_{n-1} & a_{n-\varepsilon}-\varepsilon \\
a_{n-3} & a_{n-2} & a_{n-1}
\end{array}\right| \\
M_{n}(\varepsilon)=\left|\begin{array}{lll}
a_{n}-\varepsilon & 0 & 0 \\
a_{n-1} & a_{n}-\varepsilon & 0 \\
a_{n-2} & a_{n-1} & a_{n}-\varepsilon
\end{array}\right| .
\end{gathered}
$$

Positivity of $M_{0}$ and $M_{n}(\varepsilon)$ for $0<\varepsilon<a_{n}$ is trivial. By the addition rule of determinants, we have

$$
\begin{gathered}
M_{1}=N_{1}+a_{3}\left|\begin{array}{cc}
a_{0} & a_{1} \\
0 & a_{0}
\end{array}\right| \quad M_{k}=N_{k}+a_{k+2}\left|\begin{array}{cc}
a_{k-1} & a_{k} \\
a_{k-2} & a_{k-1}
\end{array}\right| \quad k=2,3, \ldots, n-3 \\
M_{n-2}(\varepsilon)=N_{n-2}+\left(a_{n}-\varepsilon\right)\left|\begin{array}{ll}
a_{n-3} & a_{n-2} \\
a_{n-4} & a_{n-3}
\end{array}\right| .
\end{gathered}
$$

Hence

$$
M_{k}>0 \quad k=1,2, \ldots, n-3 \quad M_{n-2}(\varepsilon)>0 .
$$

Since

$$
\begin{gathered}
M_{n-1}(0)=N_{n-1}=0 \\
M_{n-1}^{\prime}(0)=2 a_{n-1} a_{n-2}-2 a_{n} a_{n-3}=2 a_{n} a_{n-2}\left(\frac{a_{n-1}}{a_{n}}-\frac{a_{n-3}}{a_{n-2}}\right)>0,
\end{gathered}
$$

we have

for sufficiently small $\varepsilon>0$.

$$
M_{n-1}(\varepsilon)>0
$$

Applying Schoenberg's test, we conclude that the sequence (4.2) is 3-times positive for all sufficiently small $\varepsilon>0$. Taking a limit as $\varepsilon$ tends to 0 , we see that the sequence $\left\{a_{k}\right\}_{k=0}^{n}=\left\{a_{0}, a_{1}, \ldots, a_{n}, 0,0, \ldots\right\}$ is 3 -times positive.

The following immediate Corollary 2 of Lemma 5 is of interest.

COROLlary 2. The bound (1.9) is sharp, and it is attained for $f(z)=a_{0} \quad\left(a_{1} z / a_{0}\right)$.

Note. It can be shown that the function $\psi(z)$ defined by (3.4) does not belong to $Q_{\infty}$ (or even to $Q_{4}$ ). Therefore the sharpness of (3.3) seems doubtful.

Lemma 6. The following equality is valid:

$$
\lim _{r \rightarrow \infty} \frac{\log M(r, \quad)}{(\log r)^{2}}=\frac{1}{2 \log c} .
$$


Proof. Since $(z) \in Q_{3} \subset P_{3}$, Theorem 1 can be applied to $(z)$. Hence

$$
\limsup _{r \rightarrow \infty} \frac{\log M(r, \quad)}{(\log r)^{2}} \leqslant \frac{1}{2 \log c} .
$$

Therefore, it suffices to prove that

$$
\liminf _{r \rightarrow \infty} \frac{\log M(r, \quad)}{(\log r)^{2}} \geqslant \frac{1}{2 \log c} .
$$

For any $n \geqslant 2$, we have

$$
\log M(r, \quad)=\log (r) \geqslant \log \frac{r^{n}}{d_{2}^{n-1} d_{3}^{n-2} \ldots d_{n}}=n \log r-\sum_{j=2}^{n}(n-j+1) \log d_{j} .
$$

Since $\lim _{j \rightarrow \infty} d_{j}=c$, we have $d_{j}<c+\varepsilon$ for any given $\varepsilon$, and $j>j_{0}=j_{0}(\varepsilon)$. Hence

$$
\begin{aligned}
\log M(r, \quad) & \geqslant n \log r-\sum_{j=2}^{j_{0}}(n-j+1) \log d_{j}-\sum_{j=j_{0}}^{n}(n-j+1) \log (c+\varepsilon) \\
& \geqslant n \log r-C_{0} n-\frac{1}{2} n^{2} \log (c+\varepsilon),
\end{aligned}
$$

where $C_{0}$ is a positive constant that depends neither on $n$ nor on $r$. Setting

we obtain

$$
n=\left[\frac{\log r}{\log (c+\varepsilon)}\right] \text {, }
$$

$$
\begin{aligned}
\log M(r,) & \geqslant\left[\frac{\log r}{\log (c+\varepsilon)}\right] \log r-C_{0}\left[\frac{\log r}{\log (c+\varepsilon)}\right]-\frac{1}{2}\left[\frac{\log r}{\log (c+\varepsilon)}\right]^{2} \log (c+\varepsilon) \\
& =\frac{(\log r)^{2}}{2 \log (c+\varepsilon)}-O(\log r) \quad r \rightarrow \infty
\end{aligned}
$$

Since $\varepsilon>0$ is arbitrarily small, we obtain (4.3).

Theorem 2 follows at once from Lemma 5 and Lemma 6 .

\section{Proof of Theorem 5}

Proof of Theorem 5(i). We shall use the following result.

THEOREM (I. J. Schoenberg [4, p. 415]). Let $g(z)=a_{0}+a_{1} z+\ldots+a_{n} z^{n}$ be $a$ polynomial with real coefficients and $a_{0}>0$. If $g(z)$ does not vanish in the angle $\{z$ : $|\arg z|<(\pi m) /(m+1)\}$, then the sequence $\left\{a_{0}, a_{1}, \ldots, a_{n}, 0,0, \ldots\right\}$ is $m$-times positive.

Applying this theorem with $m=3, g(z)=f_{n}(z)$, we obtain $f(z) \in P_{3}$. Hence, by Theorem 1, (1.1) is valid.

Note. If all sections of (0.1) do not vanish outside $\{z:|\arg z-\pi|<\pi / 4\}$, then we apply Theorem 3 or Theorem 4 instead of Theorem 1, and obtain the more precise inequalities (1.3) or (1.9) instead of (1.1).

Proof of Theorem 5(ii). We shall use the following result. 
Theorem (Hermite-Bieler [6, p. 305]). Let $P_{1}(z)$ and $P_{2}(z)$ be two polynomials with real coefficients. The polynomial

$$
\omega(z)=P_{1}(z)+\mathrm{i} P_{2}(z)
$$

does not vanish in the closed lower half-plane if and only if all zeros of $P_{1}(z)$ and $P_{2}(z)$ are simple, real, and interlacing, and, moreover, at some point $x_{0} \in \mathbf{R}$,

$$
P_{2}^{\prime}\left(x_{0}\right) P_{1}\left(x_{0}\right)-P_{2}\left(x_{0}\right) P_{1}^{\prime}\left(x_{0}\right)>0 .
$$

Assume that $f(z)$ satisfies the conditions of Theorem 5(ii). Then, for sufficiently large $n$, all zeros of

$$
f_{2 n+1}(z)=\sum_{k=0}^{2 n+1} a_{k} z^{k}
$$

lie in $\{z: \operatorname{Re} z<0\}$ so that the polynomial

$$
f_{2 n+1}(\mathrm{i} z)=\sum_{j=0}^{n} a_{2 j}(-1)^{j} z^{2 j}+\mathrm{i} z \sum_{j=0}^{n} a_{2 j+1}(-1)^{j} z^{2 j}:=p_{n}^{(1)}(z)+\mathrm{i} z p_{n}^{(2)}(z)
$$

does not vanish in the closed lower half-plane. By the Hermite-Bieler theorem, all zeros of $p_{n}^{(1)}(z)$ and $p_{n}^{(2)}(z)$ are real. Since these polynomials are even,

$$
\begin{aligned}
q_{n}^{(1)}(z) & :=p_{n}^{(1)}(\mathrm{i} \sqrt{z})=\sum_{j=0}^{n} a_{2 j} z^{j} \\
q_{n}^{(2)}(z) & :=p_{n}^{(2)}(\mathrm{i} \sqrt{z})=\sum_{j=0}^{n} a_{2 j+1} z^{j}
\end{aligned}
$$

have purely negative zeros. It means that both the formal power series

$$
q^{(1)}(z)=\sum_{j=0}^{\infty} a_{2 j} z^{j} \quad q^{(2)}(z)=\sum_{j=0}^{\infty} a_{2 j+1} z^{j}
$$

belong to $P_{\infty}$. Applying Pólya's theorem, we conclude that (5.1) converges in the whole plane and

$$
\limsup _{r \rightarrow \infty} \frac{\log M\left(r, q^{(s)}\right)}{(\log r)^{2}} \leqslant \frac{1}{2 \log 2} \quad s=1,2 .
$$

Since

we have

$$
M(r, f)=f(r)=q^{(1)}\left(r^{2}\right)+r q^{(2)}\left(r^{2}\right) \leqslant r \max _{s=1,2} M\left(r^{2}, q^{(s)}\right),
$$

$$
\limsup _{r \rightarrow \infty} \frac{\log M(r, f)}{(\log r)^{2}} \leqslant 4 \max _{s=1,2} \limsup _{r \rightarrow \infty} \frac{\log M\left(r, q^{(s)}\right)}{(\log r)^{2}} \leqslant \frac{2}{\log 2} .
$$

Note. If all sections of (0.1) do not vanish outside $\{z: \operatorname{Re} z<0\}$, we can apply (3.3) instead of (5.2) to both functions (5.1). We obtain a more complicated but more precise inequality:

$$
M(r, f) \leqslant a_{0} \psi\left(\frac{a_{2}}{a_{0}} r^{2}\right)+r a_{1} \psi\left(\frac{a_{3}}{a_{1}} r^{2}\right),
$$

where $\psi(z)$ is defined by (3.4). 
Acknowledgements. The authors thank an anonymous referee of this paper for suggestions which improved its presentation.

\section{References}

1. A. EdReI, 'Power series having partial sums with zeros in a half-plane', Proc. Amer. Math. Soc. 9 (1958) 320-324.

2. A. EdreI, E. B. SAFF and R. S. VARGa, 'Zeros of sections of power series', Lecture Notes in Mathematics 1002 (Springer, 1983) 1-115.

3. T. Ganelius, 'The zeros of the partial sums of power series', Duke Math. J. 30 (1963) 533-540.

4. S. Karlin, Total positivity - Vol. I (Stanford University Press, 1968).

5. O. Katkova and I. V. OstrovskiI, 'Zero sets of entire generating functions of Pólya frequency sequences of finite order', Math. USSR - Izv. 35 (1990) 101-112.

6. B. J. Levin, Distribution of zeros of entire functions (American Mathematical Society, Providence, 1980).

7. G. Pólya, 'Über Annäherung durch Polynome mit lauter reelle Wurzeln', Rend. Circ. Mat. Palermo 36 (1913) 279-295.

8. G. Pólya and G. Szegö, Problems and theorems in analysis - Vol. 2 (Springer, Heidelberg, 1976).

9. I. J. SCHOENBERG, 'On zeroes of generating functions of multiply positive sequences and functions', Ann. of Math. 47 (1955) 447-471.

10. E. T. Whittaker and G. N. Watson, A course of modern analysis (Cambridge University Press, 4th ed., 1990).

Department of Mathematics Bilkent University

06533 Bilkent

Ankara

Turkey

E-mail: iossif@fen.bilkent.edu.tr
B. I. Verkin Institute for Low Temperature Physics and Engineering

310164 Kharkov

Ukraine 